\title{
Mechanisms of heteroresistance to isoniazid and rifampin of Mycobacterium tuberculosis in Tashkent, Uzbekistan
}

\author{
S. Hofmann-Thiel*, J. van Ingen\#, K. Feldmann*, L. Turaev`, G.T. Uzakova ${ }^{+}$, \\ G. Murmusaeva", D. van Soolingen" and H. Hoffmann*
}

ABSTRACT: Heteroresistance of Mycobacterium tuberculosis (MTB) is defined as the coexistence of susceptible and resistant organisms to anti-tuberculosis (TB) drugs in the same patient. Heteroresistance of MTB is considered a preliminary stage to full resistance. To date, no mechanism causing heteroresistance of MTB has been proven.

Clinical specimens and cultures from 35 TB patients from Tashkent, Uzbekistan, were analysed using the Genotype MTBDR assay (Hain Lifescience, Nehren, Germany), which is designed to detect genetic mutations associated with resistance to rifampin and isoniazid. Cases of heteroresistance were further subjected to genotyping using mycobacterial interspersed repetitive unit-variable-number tandem repeat typing, spoligotyping and IS6110 fingerprinting.

Heteroresistance to rifampin and/or isoniazid was found in seven cases $(20 \%)$. In five of them, heteroresistance was caused by two different strains and in two by a single strain of the Beijing genotype. The latter cases had a history of relapse of their TB.

For the first time, two different mechanisms of heteroresistance in tuberculosis have been proven using a stepwise molecular-biological approach: 1) superinfection with two different strains, which is of interest for clinical infection control practitioners; and 2) splitting of a single strain into susceptible and resistant organisms. The latter mechanism is most likely to be related to poor treatment quality and could serve as a quality marker for tuberculosis therapy programmes in the future.

KEYWORDS: Beijing genotype, Genotype MTBDR, heteroresistance, MIRU-VNTR (mycobacterial interspersed repetitive unit-variable-number tandem repeat) typing, multidrug resistance, tuberculosis

$\mathrm{M}$ ultidrug resistance (MDR) epitomises the increasing health problem of tuberculosis (TB) in the world. According to the fourth report on the Global Project on AntiTuberculosis Drug Resistance Surveillance [1], the world's highest rate of MDR-TB (60\%) was observed in Tashkent, Uzbekistan.

MDR-TB is defined by resistance of the Mycobacterium tuberculosis (MTB) complex to at least isoniazid (INH) and rifampin (RMP). The majority of resistance to INH is caused by a mutation at codon 315 (S315T) of the kat G gene [2]. Over $95 \%$ of cases of resistance to RMP are determined by one or more mutations in an 81-bp core region of the $r p o B$ gene $[2,3]$.

Some TB patients harbour mixed populations of MTB organisms with or without resistance, a phenomenon which is referred to as heteroresistance [4]. Previous studies suggest that the relevance of heteroresistance in TB is highly underestimated [4]. To date, heteroresistance has been described for INH, RMP, ethambutol and streptomycin. It is detected using conventional drug susceptibility testing (DST) of several subcultures [5], or by simultaneous detection of wild-type (WT) and mutated sequences using PCR-based techniques, such as restriction fragment length polymorphism [6], sequencing [7] or "line probe assays" [8].

Heteroresistance of MTB is considered a preliminary stage to full resistance. Studies addressing the mechanisms underlying heteroresistance in TB are lacking so far. The aim of the present study was to analyse systematically the causes of heteroresistance to INH and RMP. The Genotype MTBDR assay (Hain Lifescience, Nehren, Germany) was used to identify such cases among TB patients of the directly observed treatment strategy (DOTS) centre in Tashkent, Uzbekistan.
AFFILIATIONS

*IML, Institute of Microbiology \& Laboratory Medicine, Supranational Reference Laboratory of Tuberculosis, Gauting, Germany. ${ }^{\#}$ National Mycobacteria Reference Laboratory, National Institute of Public Health and the Environment, Bilthoven, The Netherlands.

'National Reference Laboratory of Tuberculosis, TB-Institute, and ${ }^{+}$DOTS Center, Global Fund to Fight AIDS, Tuberculosis and Malaria, Tashkent, Uzbekistan.

\section{CORRESPONDENCE}

H. Hoffmann

IML, Institute of Microbiology \& Laboratory Medicine, Supranational Reference Laboratory (SNRL),

Asklepios Fachkliniken, Robert-KochAllee 2, D-82131 Gauting, Germany. Fax: 4989857918350

E-mail: Harald.Hoffmann@

asklepios.com

Received:

June 132008

Accepted after revision:

September 232008

SUPPORT STATEMENT

Drug susceptibility testing was performed with financial support from KfW Bankengruppe (Frankfurt, Germany) and the Global Fund to Fight AIDS, Tuberculosis and Malaria (Geneva, Switzerland) in the frame of the Drug Resistance Survey of Uzbekistan

\section{STATEMENT OF INTEREST}

A statement of interest for this study can be found at

www.erj.ersjournals.com/misc/ statements.shtml

European Respiratory Journal Print ISSN 0903-1936

Online ISSN 1399-3003 


\section{MATERIALS AND METHODS}

\section{Clinical specimens}

Sputa of 35 pulmonary TB patients attending the DOTS centre in Tashkent were collected for the present study. These were sent to the supranational reference laboratory (SNRL) in Gauting, Germany, for DST in the frame of the Drug Resistance Survey in the city. The sputa comprised consecutive samples which were processed on February 10 (cases 11691185), February 22 (cases 1565-1579) and May 15, 2006 (cases 4070-4072). The investigator ( $\mathrm{H}$. Hoffmann) processing the samples was not aware of the clinical history of the patients. All sputa were spot specimens. They were produced spontaneously and collected under observation.

The clinical data and treatment history of patients are summarised in table 1. Patients' data were kept anonymous. The study was approved ethically by the Ministry of Health, Tashkent, Uzbekistan.

\section{Bacterial isolates}

With few modifications, sputa were processed as recommended by the International Union Against Tuberculosis and Lung Disease [9] using decontamination by the $N$-acetylcysteine- $\mathrm{NaOH}$ method. After inoculation for growth detection, the leftover sediment was used for Genotype MTBDR testing. When liquid cultures turned positive, isolates were sub-cultured and DST was performed for first-line drugs including INH and RMP in Mycobacteria Growth Indicator Tubes (MGIT ${ }^{\mathrm{TM}}$; Becton-Dickinson, Heidelberg, Germany) in the BACTEC $^{\mathrm{TM}}$ MGIT 960 incubator (Becton-Dickinson) following the protocol of the manufacturer.

Decontaminated specimens or cultured bacteria were inactivated at $100^{\circ} \mathrm{C}$ for $20 \mathrm{~min}$ followed by sonication. The suspension was centrifuged and the supernatant used for PCR. Amplification and sequencing of the kat $G$ and rpoB loci were performed using standard protocols with primers rpoB-f (5'-GGG AGC GGA TGA CCA CCC A-3'), rpoB-r (5'-GCG GTA CGG CGT TTC GAT GAA C-3'), katG-f (5'-CGG CGC ATG GCC ATG AAC GAC GTC-3') and katG-r (5'-CCG GCA CCG GCG CCG TCC TTG-3').

\section{Genotype MTBDR assay}

The Genotype MTBDR assay was carried out according to the manufacturer's recommendations using $5 \mu \mathrm{L}$ of DNA extracts and Taq polymerase (Invitrogen, Karlsruhe, Germany). The test detects gene mutations in the rpoB and $k a t G$ genes and is based on multiplex PCR followed by reverse hybridisation of amplicons to respective WT and mutation probes. PCR cycling using DNA extracted from sputa was adapted to the optimised method of BANG et al. [10].

\section{Typing of MTB}

Molecular typing of MTB was based on 24 different loci containing variable numbers of tandem repeats (VNTR) of mycobacterial interspersed repetitive units (MIRU) [11]. Multiplex PCRs and automated MIRU-VNTR analyses were performed as previously described [11, 12]. Extraction of genomic DNA from mycobacteria and IS6110 fingerprinting were carried out according to the standardised protocol described elsewhere [13]. Spoligotyping was performed according to KAMERBEEK et al. [14].

\section{RESULTS}

\section{Phenotypic and genotypic DST}

According to conventional DST, 13 (37\%) of the 35 isolates were MDR (INHr, RMPr), 10 (28\%) were resistant to INH (INHr, RMPs) and 12 (34\%) were susceptible to INH and RMP (INHs, RMPs; table 2). There were no cases of resistance to RMP only (INHs, RMPr).

Genotype MTBDR was applied directly to decontaminated sputa and later to the bacterial cultures. When applied to cultures, 19 isolates (54\%) were detected with mutations in the kat $G$, and $14(40 \%)$ with mutations in the rpoB gene (table 2). Test results from the sputa were concordant with those from the cultures in all but three cases, which were subsequently identified as heteroresistant to INH or RMP. In four cases, rpoB-specific hybridisation signals were markedly weaker, yielding indeterminate test results.

\section{Heteroresistance to INH and/or RMP}

In seven cases (20\%), there was evidence of heteroresistance to either INH or RMP (fig. 1). In three cases with resistance to

TABLE 1 Patient descriptions: clinical data and treatment history

\begin{tabular}{|c|c|c|c|}
\hline \multirow[t]{2}{*}{ Clinical data } & \multirow[t]{2}{*}{ Patients n (\%) } & \multicolumn{2}{|c|}{ Treatment history } \\
\hline & & Before sputum collection & After sputum collection \\
\hline New cases & $21(60)$ & No treatment & $\begin{array}{c}\text { 2-3 months: INH/RMP/PZA/EMB } \\
4 \text { months: INH/RMP }\end{array}$ \\
\hline Treatment failure & $8(23)$ & Treatment like "new cases" & $\begin{array}{c}\text { After diagnosis of "treatment failure": } \\
2 \text { months: INH/RMP/PZA/EMB/SM } \\
1-2 \text { months: INH/RMP/PZA/EMB } \\
5 \text { months: INH/RMP/EMB }\end{array}$ \\
\hline Relapse & $6(17)$ & Treatment like "new cases" & $\begin{array}{l}\text { After diagnosis of "relapse": } \\
\text { treatment like cases with "treatment failu }\end{array}$ \\
\hline
\end{tabular}

The total number of patients was $n=35$. INH: isoniazid; RMP: rifampin; PZA: pyrazinamide; EMB: ethambutol; SM: streptomycin. ${ }^{*}$ : treatment according to the directly observed treatment strategy protocol. 


\begin{tabular}{lccc} 
TABLE 2 & Phenotypic and genotypic susceptibility testing \\
& DST in MGIT & \multicolumn{2}{c}{ Genotype MTBDR } \\
\cline { 3 - 4 } & & Cultures $^{\#}$ & Sputum \\
\hline INHs, RMPs & $12(34)$ & $14(40)$ & $13(37)$ \\
INHr, RMPr & $13(37)$ & $12(34)$ & $9(26)$ \\
INHr, RMPs & $10(28)$ & $7(20)$ & $7(20)$ \\
INHs, RMPr & $0(0)$ & $2(6)$ & $2(6)$ \\
Not interpretable & $0(0)$ & $0(0)$ & $4(11)$ \\
\hline
\end{tabular}

The total number of samples was $n=35$. Data are presented at $n$ (\%). DST: drug susceptibility testing; MGIT: Mycobacterial Growth Indicator Tube; INHs: susceptible to isoniazid (INH); RMPs: susceptible to rifampin (RMP); $\mathbb{I N H}$ :

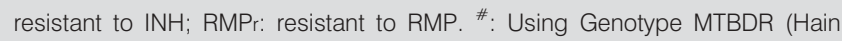
Lifescience, Nehren, Germany), the following mutations were identified: 19 cases with mutations in katG, of whom 18 had mutation S315T and one a missing katG wild-type band; 14 cases with mutations in rpoB, eight of them with S531L, two with D516V, and one with H526Y and three with missing rpoB wild-type bands.
INH (1171, 1571 and 4071), the katG WT and the S315T-specific hybridisation bands were visible simultaneously. In two of them (4071 and 1571), heteroresistance was visible in both sputa and cultures and in one (1171) it was seen only after culturing.

In five cases, $г р о B$ hybridisation patterns indicated heteroresistance to RMP (fig. 1); one of them (case 4071) simultaneously showed heteroresistance to INH. In cases 1567, 4071 and 4072, heteroresistance to RMP was evident both in sputum and culture. In case 1177, the WT band and two mutation bands (S531L plus H526Y) were detected in the sputum, consistent with the coexistence of three different organisms. The S531L-specific band disappeared after culturing. In case 1575 , all rроB WT signals were detectable in sputum whereas WT band 3 (codons 521-525) disappeared in culture, suggesting overgrowth of the WT by mutated organisms. In all seven cases of heteroresistance to either drug, the results of phenotypic DST corresponded to the mutated, i.e. resistant, organism.

\section{Verification of heteroresistance}

The coexistence of WT and mutated sequences was confirmed by sequencing of the rpoB and katG PCR products for all seven

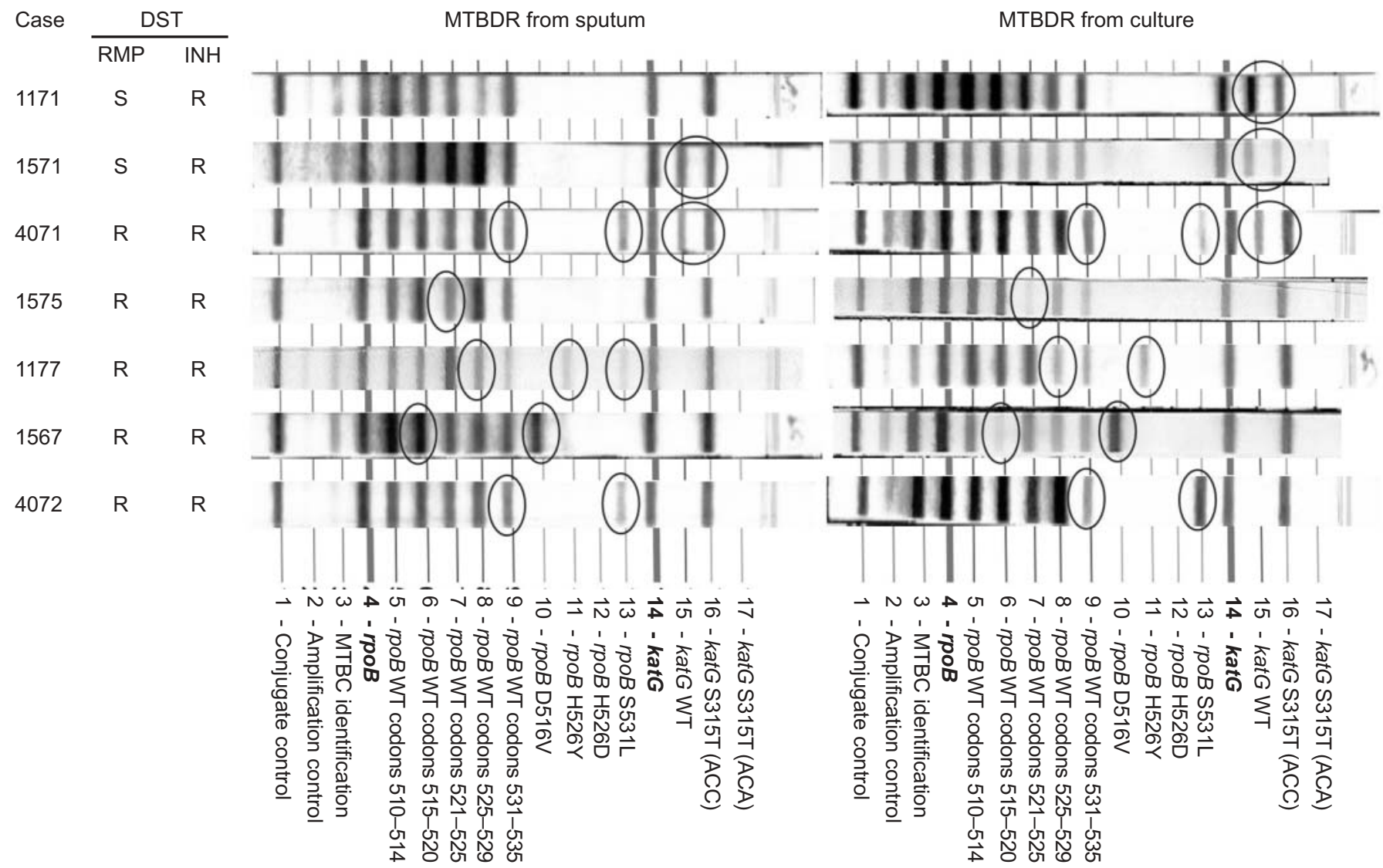

FIGURE 1. Heteroresistance in seven tuberculosis patients as detected by Genotype MTBDR (Hain Lifescience, Nehren, Germany). Genotype MTBDR was applied directly to the sputum and later to cultures. Simultaneous detection of wild-type (WT) and mutation-specific bands for katG and/or rpoB became visible in all seven cases (circled).The missing WT band in case 1575 corresponded to mutation S522L as shown by sequencing. The results of conventional drug susceptibility testing (DST) are indicated. RMP: rifampin; INH: isoniazid; S: susceptible; R: resistant; MTBC: Mycobacterium tuberculosis complex. 
a)

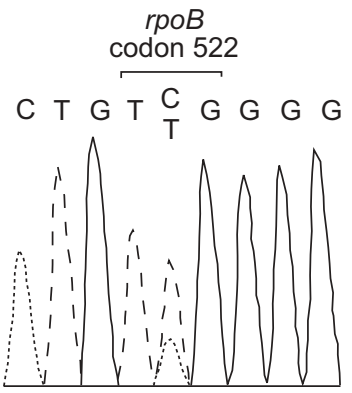

b)

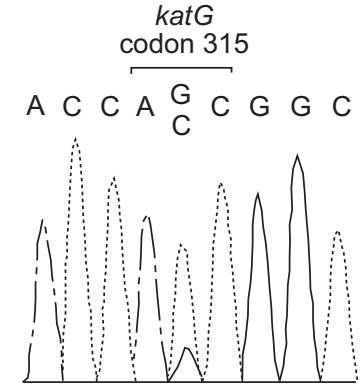

c) Ratio H37 : MDR

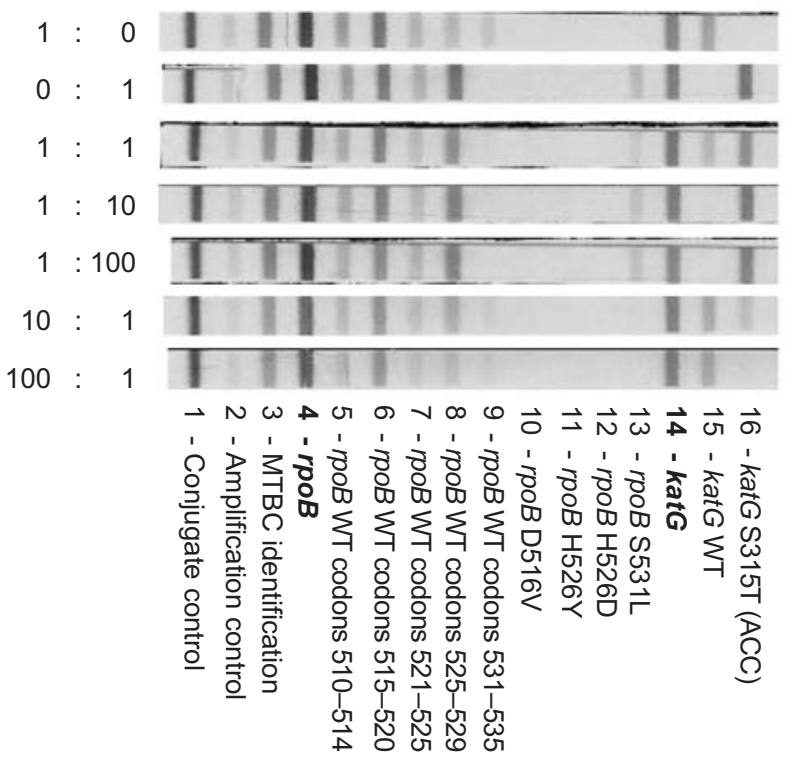

FIGURE 2. Tests for specificity of Genotype MTBDR (Hain Lifescience, Nehren, Germany) results. a and b) Heteroresistance was confirmed by sequencing. Example sequencing results showing a) both the wild-type (WT) sequence (TCG) and the mutation (TTG) at codon 522 of rpoB (case 1575) as well as b) WT (AGC) and mutation (ACC) at codon 315 of katG (case 1571). c) Artificial heteroresistance was generated using different mixtures of the susceptible reference strain H37Rv (sensitive to rifampin (RMP) and isoniazid $(\mathrm{INH})$ ) and a multidrug-resistant (MDR) strain (resistant to RMP and INH) derived from the supranational reference laboratory quality-assessment strain collection. The mixtures were tested with the Genotype MTBDR. MTBC: Mycobacterium tuberculosis complex.

cases, respectively (fig. $2 \mathrm{a}$ and b). In order to check for reproducibility of hybridisation patterns, the Genotype MTBDR assay was repeated and supplemented with the Genotype MTBDRplus with all seven sputa and cultures from the cases with heteroresistance. The results were concordant with the first ones (data not shown). Furthermore, analysis of 64 MTB cultures of the SNRL quality-assessment strains with the Genotype MTBDR did not show cases of heteroresistance (table 3). It is therefore unlikely that simultaneous detection of WT and mutation bands was due to nonspecific hybridisation.

To assess the relative proportions of WT and mutated organisms needed to allow for the simultaneous visualisation of WT and mutation signals, artificial heteroresistance was produced using different mixtures of susceptible and resistant bacteria followed

\begin{tabular}{lcc} 
TABLE 3 & $\begin{array}{l}\text { Genotype MTBDR results using } 64 \text { strains of the } \\
\text { supranational reference laboratory external } \\
\text { quality assessment }\end{array}$ \\
& Genotype MTBDR & DST \\
\hline & 20 & 15 \\
\hline INHs & 44 & 49 \\
INHr & 0 & 64 \\
Heteroresistance & 64 & 33 \\
Total & 27 & 31 \\
RMPs & 37 & 64 \\
RMPr & 0 & \\
Heteroresistance & 64 & \\
Total & \\
\hline & \\
Data are presented as n. DST: drug susceptibility testing; INHs: susceptible to \\
isoniazid (INH); INHr: resistant to INH; RMPs: susceptible to rifampin (RMP); \\
RMPs: resistant to RMP.
\end{tabular}

by DNA extraction and Genotype MTBDR. WT and mutation bands were simultaneously visible at ratios of the WT to the resistant strain of 1:1, 10:1 and 1:10 (fig. 2c). The hybridisation signal of the lower-concentration strain disappeared when the ratio was 1:100 or lower. Thus, Genotype MTBDR seems to be a reliable and specific method to detect heteroresistance of MTB, provided that the relative proportion of the organisms is $\geqslant 10 \%$.

\section{Characterisation of heteroresistant MTB isolates}

To distinguish whether the occurrence of heteroresistance to INH and/or RMP originated from infection with different MTB strains or from infection with a single strain separated into two lineages of organisms, MIRU-VNTR typing was applied to sputum samples and cultures. In case of infection with two strains, two distinct MTB genotypes should be detectable in sputum and/or culture. In four cases of heteroresistance (1171, 1571, 1575 and 4071), two distinct alleles were detected simultaneously at two or more loci (table 4) suggesting the presence of two different genotype strains. In cases 1177, 1567 and 4072, only single genotypes were seen.

In order to estimate the relevance of the Beijing genotype among cases of heteroresistance, spoligotyping was performed from cultures. Beijing genotype strains are predominantly found in Asia and the former Soviet Union [15]. Spoligotyping of cases 1177, 1567 and 4072 yielded patterns corresponding to the Beijing genotype (fig. 3a). Spoligo-patterns of cases 1171, 1571 and 4071 could not be assigned to known patterns but showed a mixture of strong and faint signals, consistent with mixtures of two different strains.

Although MIRU-VNTR typing suggested the presence of single strains in the cases with Beijing genotypes, the presence of two different Beijing genotype strains could not be excluded. Therefore, IS6110 fingerprinting of cultures grown either in the absence or in the presence of RMP was performed. In case of infection with two Beijing strains, different patterns should be obtained from the resistant organisms selected on RMP and the mixed organisms grown in drug-free medium. IS6110 typing gave identical patterns with strains 1177 and 1567 but slight 


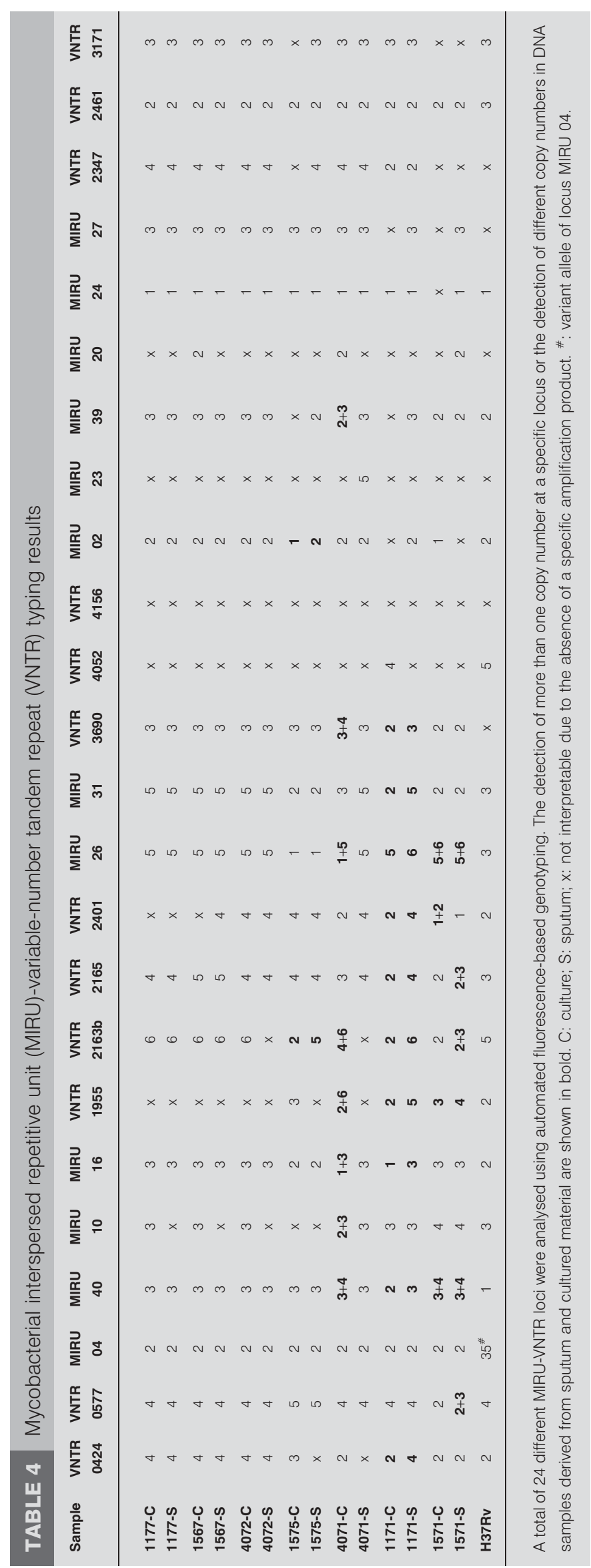

differences with strain 4072 (fig. 3b) suggesting infection with two different Beijing strains in the latter case.

Evaluation of clinical data showed that in three cases of heteroresistance, TB had been newly diagnosed whereas four cases had a history of treatment failure or relapse (table 5). Comparison of biological and clinical data showed that all "new cases" were infected with two different strains. Both cases with single strains were "relapses". Notably, all three cases with Beijing genotype strains showed a history of relapse, whereas among the four non-Beijing cases, three were new and one had experienced "treatment failure" (table 5). Thus, the risk of relapse or treatment failure was higher in cases with Beijing genotype strains.

\section{DISCUSSION}

In the present study, molecular investigation of 35 TB patients from Tashkent, identified seven cases $(20 \%)$ of heteroresistance. For the first time, two mechanisms of heteroresistance were proven, i.e. the coexistence of two different MTB strains and the segregation of single strains into resistant and susceptible organisms. These mechanisms were linked to the clinical entities new cases, treatment failures and relapses. The present study showed that the coexistence of two different strains prevailed in new cases, while segregation of single strains prevailed in treatment failures and relapses.

Heteroresistance due to infection with two different strains is theoretically explained by superinfection of a patient already infected with one MTB strain with an additional one. For the three new cases, superinfection of a latent TB infection seemed to be the best explanation, although the hypothesis that superinfection can trigger a reactivation of pre-existing latent TB has so far never been proven [16]. In contrast, superinfection of patients with active TB has repeatedly been demonstrated, particularly with resistant Beijing genotype strains $[17,18]$. This mechanism could explain the heteroresistance due to infection with two strains in patients with a history of relapse or treatment failure.

A high TB incidence certainly increases the risk of superinfection. Uzbek TB hospitals might be risky sites of superinfection with resistant strains. This will be investigated in future studies and may give valuable information for infectioncontrol practitioners.

Heteroresistance due to infection with single strains is most probably explained by segregation into susceptible and resistant organisms under the selective pressure of insufficient anti-TB therapy. Numerous reports have described the evolution of resistance due to inadequate therapy [19]. Ineffective therapy can result from noncompliance of the patient, poor pharmaceutical quality of the drugs, or pre-existing resistances of the pathogens. Notably, both patients with heteroresistance due to single strains had a history of relapse. Thus, positive selective pressure of inadequate therapy could have amplified the resistant organisms to proportions detectable by the Genotype MTBDR assay. If so, the rate of heteroresistance with single strains could serve as an indicator for the quality of anti-TB treatment programmes, which could be of help for public health practitioners.

In the current study, heteroresistance due to infection with single strains was caused exclusively by Beijing genotype 
a)

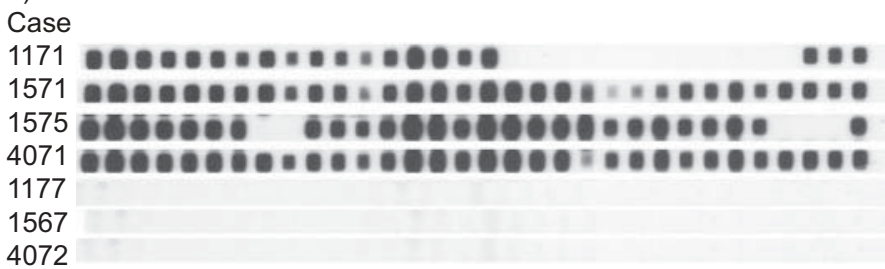

b)

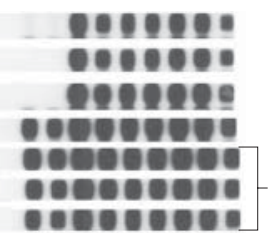

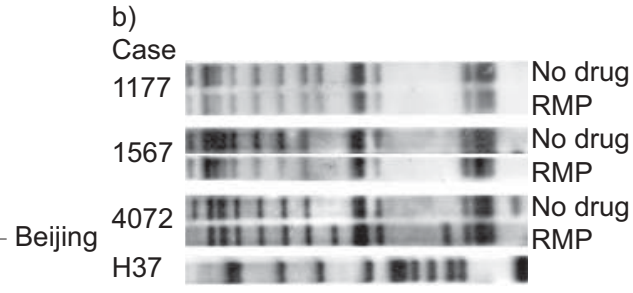

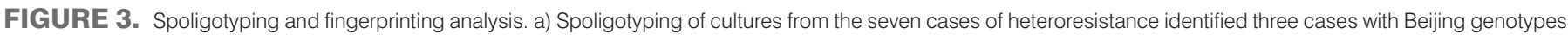

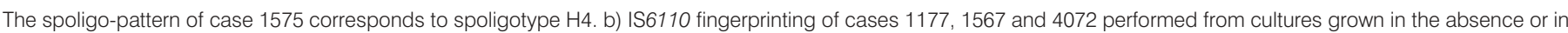
the presence of rifampin (RMP). As control, the IS6110 fingerprint from strain H37Rv is shown.

TABLE 5 Typing results and clinical data of the seven cases with heteroresistant tuberculosis

\begin{tabular}{llcc} 
Sample & Infection with single or two strains & Genotype(s) & Clinical data \\
\hline $\mathbf{1 1 7 7}$ & Single strain & Beijing & Relapse \\
$\mathbf{1 5 6 7}$ & Single strain & Beijing & Relapse \\
$\mathbf{4 0 7 2}$ & Two strains & Beijing/Beijing & Relapse \\
$\mathbf{1 5 7 5}$ & Two strains & H4/unknown & Treatment failure \\
$\mathbf{4 0 7 1}$ & Two strains & Unknown/unknown & New case \\
$\mathbf{1 1 7 1}$ & Two strains & Unknown/unknown & New case \\
$\mathbf{1 5 7 1}$ & Two strains & Unknown/unknown & New case \\
\hline
\end{tabular}

Data from mycobacterial interspersed repetitive unit-variable-number tandem repeat typing, spoligotyping and fingerprinting were summarised and compared with clinical data. ${ }^{\#}$ : case 4071 had a spoligo-pattern that was potentially consistent with a mixture of a Beijing genotype plus a non-Beijing strain.

strains. Single nucleotide polymorphisms in mismatch-repair genes enable the Beijing genotype to acquire resistanceassociated mutations more easily than other genotypes [20]. This observation and the predominance of the Beijing genotype in Central Asian countries might explain why only Beijing genotype strains have been observed in the present patient group. Furthermore, heteroresistance due to infection with Beijing strains was invariably linked to the history of relapse. This is in line with previous studies reporting an association between relapses or treatment failures and the Beijing genotype in Vietnam [21] and Singapore [22].

The rate of $20 \%$ of heteroresistance in $\mathrm{TB}$ is similar to the finding by RINDER et al. [6], who reported a rate of $17 \%$. Other studies have reported significantly lower rates $[8,23,24]$. This discrepancy may depend on several factors. First, the group of TB patients and the study site influence the rate of heteroresistance. Among the current TB patients, a high rate of MDRTB was observed and $40 \%$ had previous failure of therapy or relapses. Similarly, in the study of RINDER et al. [6], only DNA samples from patients with risk factors such as prior anti-TB treatment were included. Secondly, application of assays directly to the clinical specimens enhances the chance of detecting heteroresistance [4, 24]. Thirdly, the simultaneous testing of clinical specimens and bacterial cultures further improves the detection of heteroresistance.

In conclusion, to date heteroresistance is an underestimated phenomenon in tuberculosis, especially in highly endemic areas. In the current study, heteroresistance was primarily caused by co-infection with different Mycobacterium tuberculosis strains, certainly favoured by the high incidence of tuberculosis in Tashkent. Further studies with larger numbers of patients are needed to estimate the epidemiology and the clinical impact of heteroresistance.

\section{ACKNOWLEDGEMENTS}

The current authors would like to thank L. Slutzka (IML, Gauting, Germany) and A. van den Brandt (National Mycobacteria Reference Laboratory, Bilthoven, The Netherlands) for their excellent technical assistance.

\section{REFERENCES}

1 World Health Organization. Anti-tuberculosis Drug Resistance in the World. Report No. 4. The WHO/ IUATLD Global Project on Anti-tuberculosis Drug Resistance Surveillance 2002-2007. Geneva, World Health Organization, 2008.

2 Ramaswamy S, Musser JM. Molecular genetic basis of antimicrobial agent resistance in Mycobacterium tuberculosis: 1998 update. Tuber Lung Dis 1998; 79: 3-29.

3 Heep M, Brandstatter B, Rieger U, et al. Frequency of rpoB mutations inside and outside the cluster $I$ region in rifampin-resistant clinical Mycobacterium tuberculosis isolates. J Clin Microbiol 2001; 39: 107-110.

4 Rinder H. Hetero-resistance: an under-recognised confounder in diagnosis and therapy? J Med Microbiol 2001; 50: 1018-1020.

5 Adjers-Koskela K, Katila ML. Susceptibility testing with the manual mycobacteria growth indicator tube (MGIT) and the MGIT 960 system provides rapid and reliable 
verification of multidrug-resistant tuberculosis. I Clin Microbiol 2003; 41: 1235-1239.

6 Rinder H, Mieskes KT, Loscher T. Heteroresistance in Mycobacterium tuberculosis. Int J Tuberc Lung Dis 2001; 5: 339-345.

7 Karahan ZC, Akar N. Restriction endonuclease analysis as a solution for determining rifampin resistance mutations by automated DNA sequencing in heteroresistant Mycobacterium tuberculosis strains. Microb Drug Resist 2005; 11: 137-140.

8 de Oliveira MM, da Silva Rocha A, Cardoso Oelemann M, et al. Rapid detection of resistance against rifampicin in isolates of Mycobacterium tuberculosis from Brazilian patients using a reverse-phase hybridization assay. J Microbiol Methods 2003; 53: 335-342.

9 International Union Against Tuberculosis and Lung Disease. Priorities for Tuberculosis Bacteriology Services in Low-income Countries. 2nd Edn. Paris, International Union Against Tuberculosis and Lung Disease, 2007.

10 Bang D, Bengard Andersen A, Thomsen VO. Rapid genotypic detection of rifampin- and isoniazid-resistant Mycobacterium tuberculosis directly in clinical specimens. J Clin Microbiol 2006; 44: 2605-2608.

11 Supply P, Allix C, Lesjean S, et al. Proposal for standardization of optimized mycobacterial interspersed repetetive unitvariable-numer of tandem repeat typing of Mycobacterium tuberculosis. J Clin Microbiol 2006; 44: 4498-4510.

12 Supply P, Lesjean S, Savine E, Kremer K, van Soolingen D, Locht C. Automated high-throughput genotyping for study of global epidemiology of Mycobacterium tuberculosis based on mycobacterial interspersed repetetive units. J Clin Microbiol 2001; 39: 3563-3571.

13 van Embden JD, Cave MD, Crawford JT, et al. Strain identification of Mycobacterium tuberculosis by DNA fingerprinting: recommendations for a standardized methodology. J Clin Microbiol 1993; 31: 406-409.

14 Kamerbeek J, Schouls L, Kolk A, et al. Simultaneous detection and strain differentiation of Mycobacterium tuberculosis for diagnosis and epidemiology. I Clin Microbiol 1997; 35: 907-914.

15 van Soolingen D, Quian L, de Haas PEW, et al. Predominance of a single genotype of Mycobacterium tuberculosis in countries of East Asia. J Clin Microbiol 1995; 33: 3234-3238.

16 Kaufmann SHE, McMichael AJ. Anulling a dangerous liaison: vaccination strategies against AIDS and tuberculosis. Nat Med 2005; 11: Suppl. 4, S33-S44.

17 Warren RM, Victor TC, Streicher EM, et al. Patients with active tuberculosis often have different strains in the same sputum specimen. Am J Respir Crit Care Med 2004; 169: 610-614.

18 Cox HS, Orozco JD, Male R, et al. Multidrug-resistant tuberculosis in Central Asia. Emerg Infect Dis 2004; 10: 865-872.

19 Woodford N, Ellington MJ. The emergence of antibiotic resistance by mutation. Clin Microbiol Infect 2006; 13: 5-18.

20 Rad ME, Bifani P, Martin C, et al. Mutations in putative mutator genes of Mycobacterium tuberculosis strains of the W-Beijing family. Emerg Infect Dis 2004; 9: 838-845.

21 Lan NT, Lien HT, Tung le B, Borgdorff MW, Kremer K, van Soolingen D. Mycobacterium tuberculosis Beijing genotype and risk for treatment failure and relapse, Vietnam. Emerg Infect Dis 2003; 9: 1633-1635.

22 Sun YJ, Lee AS, Wong SY, Paton NI. Association of Mycobacterium tuberculosis Beijing genotype with tuberculosis in Singapore. Epidemiol Infect 2006; 134: 329-332.

23 Mäkinen J, Marttila HJ, Marjamäki M, Viljanen MK, Soini H. Comparison of two commercially available DNA line probe assays for detection of multidrug-resistant Mycobacterium tuberculosis. J Clin Microbiol 2006; 44: 350-352.

24 Miotto P, Piana F, Penati V, Canducci F, Migliori GB, Cirillo DM. Use of genotype MTBDR assay for molecular detection of rifampin and isoniazid resistance in Mycobacterium tuberculosis clinical strains isolated in Italy. J Clin Microbiol 2006; 44: 2485-2491. 